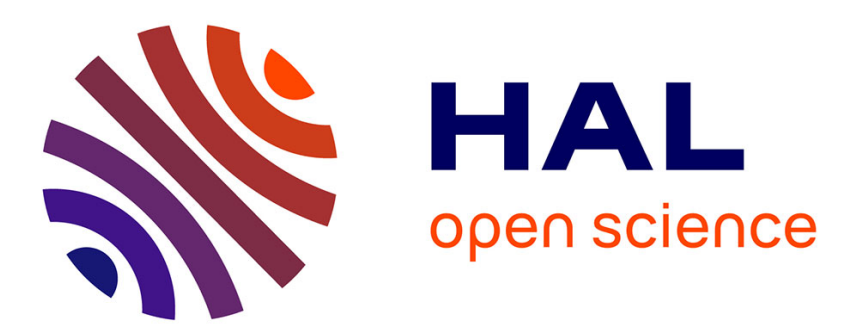

\title{
Occurrence of the magnetic polarization on an iron adatom on a matrix of transition metals of the first row
}

\author{
G. Allan, P. Lenglart
}

\section{To cite this version:}

G. Allan, P. Lenglart. Occurrence of the magnetic polarization on an iron adatom on a matrix of transition metals of the first row. Journal de Physique, 1974, 35 (1), pp.91-95. 10.1051/jphys:0197400350109100 - jpa-00208129

HAL Id: jpa-00208129

https://hal.science/jpa-00208129

Submitted on 1 Jan 1974

HAL is a multi-disciplinary open access archive for the deposit and dissemination of scientific research documents, whether they are published or not. The documents may come from teaching and research institutions in France or abroad, or from public or private research centers.
L'archive ouverte pluridisciplinaire HAL, est destinée au dépôt et à la diffusion de documents scientifiques de niveau recherche, publiés ou non, émanant des établissements d'enseignement et de recherche français ou étrangers, des laboratoires publics ou privés. 


\title{
OCCURRENCE OF THE MAGNETIC POLARIZATION ON AN IRON ADATOM ON A MATRIX OF TRANSITION METALS OF THE FIRST ROW
}

\author{
G. ALLAN and P. LENGLART \\ Laboratoire de Physique des Solides (*), ISEN, 3, rue François-Baës, \\ 59046 Lille Cedex, France
}

(Reçu le 28 mai 1973, révisé le 14 septembre 1973)

\begin{abstract}
Résumé. - On utilise un modèle simple introduit par Clogston [1] pour étudier l'existence d'une polarisation magnétique sur un atome de fer dans différents environnements. On discute le cas d'un adatome et celui d'un atome en position de substitution dans une surface comparé au cas bien connu d'une impureté de substitution en volume. On montre que la polarisation magnétique apparaît plus facilement lorsque l'on passe du cas d'une impureté de substitution en volume à celui d'une impureté de substitution dans le plan de surface et de la même façon lorsque l'on passe de ce dernier cas à celui d'un adatome. Des résultats expérimentaux sont attendus pour développer un modèle plus élaboré.
\end{abstract}

\begin{abstract}
We use a simple model developed by Clogston [1] to study the occurrence of magnetic polarization on an iron atom in different surroundings. We discuss the cases of an adatom or a substitutional impurity. It is shown that the polarization appears more easily when an atom of iron goes from a bulk substitutional place to a surface substitutional place and in a same way when it goes from this place to an adsorbed place. Experimental results are awaited for a more elaborate theoretical study.
\end{abstract}

1. Introduction. - Some years ago susceptibility measurements [1] showed the existence of a localized magnetic moment on an iron atom impurity in several bulk transition metals of the second row. The study of the occurrence of a magnetic moment on an iron impurity has been fully developed [2] ... [6]. The magnetic polarization arises from the electron-electron exchange potential. The most important matrix element of that potential turns to be the Slater-Stoner term $U n_{\mathrm{d} \uparrow} n_{\mathrm{d} \downarrow}$ [7]. We here follow the presentation of Wolff [5]. Many other studies [6] have since then been done to include the correlations and to take into account the polarization of the atoms close to the iron impurity. We only want to show in a crude model that such a magnetic polarization may appear still more easily on an iron atom adsorbed on a surface of transition metal.

This model, which is a Hartree-Fock approximation, may only be significant at low temperature, however not too low in order to neglect the magnetic fluctuations and the Kondo effect [8]. The increase of the local density in the case of an adatom compared to the local density of a bulk impurity may induce an instability of the parametric state. To study this possibility we use the Stoner condition which shows

$\left(^{*}\right)$ Equipe de recherche du CNRS. that the paramagnetic state would be instable compared to the magnetic one but does not consider the case of metastability. We only then consider the conditions of appearance of the magnetic instability. No attempt will be made to describe the actual state. Inside this model, we consider only the case of a transitional matrix of the first row.

2. Approximations on the band-structure of the transition metals. - In this section we introduce an approximative « $\mathrm{d}$ » band structure for the transition metal using a tight binding approximation. We define a density of states $n_{0}(E)$ for the matrix and a local density $n_{\mathrm{L}}(E)$ only using its first moments. In order to simplify the calculation, the density of states and the local density will be chosen fivefold degenerate. We shall take the resonance integrals between the iron atom and its neighbours equal to the resonance integrals of the bulk. The only difference inside this model between the iron atom and a bulk atom lies in the filling up of the local density. The local density on an iron bulk impurity is then the density $n_{0}(E)$ of the transitional matrix. In the one-electron approximation the hamiltonian is given by :

$$
\mathscr{H}_{0}=\frac{p^{2}}{2 m}+\sum_{j} V\left(r-R_{j}\right)
$$


where the summation is extended to the whole perfect infinite body centered cubic crystal or to the crystal with a surface. We neglect the s band and in a tightbinding approximation the resonance integrals between first and second nearest neighbours can be expressed in terms of the $\mathrm{dd} \sigma, \mathrm{dd} \pi$ and $\mathrm{dd} \delta$ SlaterKoster integrals [9]. The ratios of these integrals are almost independent of the transition metal [10]. So we take these ratios equal to the values deduced from an interpolation scheme of the $\mathrm{W}$ band structure calculated by Mattheiss [11]. The width of the $d$ band is adjusted to the reasonable value of $6 \mathrm{eV}$ for the first transition metal row [12]. This adjustment is of less importance because each integral can be expressed in terms of the band width if the ratios $\mathrm{dd} \pi / \mathrm{dd} \sigma$ and $\mathrm{dd} \delta / \mathrm{dd} \sigma$ are known, it is only made to give an idea of the resonance integral values (Table I).

\section{TABLE I}

\section{Resonance integrals $(\mathrm{eV})$}

$\begin{array}{lcc} & \text { 1st neighbour } & \text { 2nd neighbour } \\ \mathrm{dd} \sigma & -\overline{0.060} & -\overline{0.035} \\ \mathrm{dd} \pi & 0.031 & 0.020 \\ \mathrm{dd} \delta & -0.003 & -0.002\end{array}$

In the same way, we introduce two shift integrals $d_{0}$ and $d_{0}^{\prime}$ defined by Slater and Koster as :

$$
\begin{aligned}
& d_{0}=\left\langle x y\left|\sum_{j \neq 0} V\left(r-R_{j}\right)\right| x y\right\rangle \\
& d_{0}^{\prime}=\left\langle x^{2}-y^{2}\left|\sum_{j \neq 0} V\left(r-R_{j}\right)\right| x^{2}-y^{2}\right\rangle
\end{aligned}
$$

whose difference may be evaluated as $0.025 \mathrm{eV}$.

As the problem of the surface electronic structure is still difficult even in such a simple tight-binding approximation for a $\mathrm{d}$ band, we have made other approximations to get the surface density of states. In each case we consider below, we treat the $d$ band as a five fold degenerate band and we write the density of states $n_{0}(E)$ or the local density $n_{\mathrm{L}}(E)$ as :

$$
n_{0}(E)=A\left(E^{4}-E_{0}^{4}\right)+B\left(E^{2}-E_{0}^{2}\right)
$$

where $A, B$ and $E_{0}$ are fitted to the first significant moments of the state density, e. g. $\mu_{0}, \mu_{2}$ and $\mu_{4}$. Let us recall that

$$
\mu_{n}=\int E^{n} n_{0}(E) \mathrm{d} E .
$$

We have :

$$
\begin{aligned}
& E_{0}=\left[7 \mu_{2}-\left(49 \mu_{2}^{2}-21 \mu_{4}\right)^{1 / 2}\right]^{1 / 2} \\
& A=-\frac{105}{32 E_{0}^{7}}\left(5 \mu_{2}-E_{0}^{2}\right) \\
& B=\frac{105}{16 E_{0}^{5}}\left(3 \mu_{2}-\frac{5}{7} E_{0}^{2}\right) .
\end{aligned}
$$

The moments calculated by a random walk method [13] are given in table II for a bulk, surface or adsorbed atom (same transition adatom as the bulk atoms). In each case, $\mu_{0}$ is equal to unity.

TABLE II

Values of the moments

$\begin{array}{lccc} & \text { Bulk } & \text { Surface } & \text { Adatom } \\ \mu_{2}(\mathrm{eV})^{2} & \overline{2.14} & \overline{1.25} & \overline{0.91} \\ \mu_{4}(\mathrm{eV})^{4} & 7.43 & 3.25 & 1.82\end{array}$

In the case of a bulk atom with our values of resonance integrals, $\mu_{1}, \mu_{3}$ and $\mu_{5}$ are respectively equal to $0.14 \mathrm{eV}, 0.23(\mathrm{eV})^{3}$ and $0.47(\mathrm{eV})^{5}$. Since they are small a symmetric shape of the density of states will not alter our results. The shapes of the densities of states are also smoothed (Fig. 1) and their behaviour with $\mu_{2}$ can be compared with the results of Kalkstein and Soven for a simple cubic lattice [14]. However it is interesting to see that such a crude expression

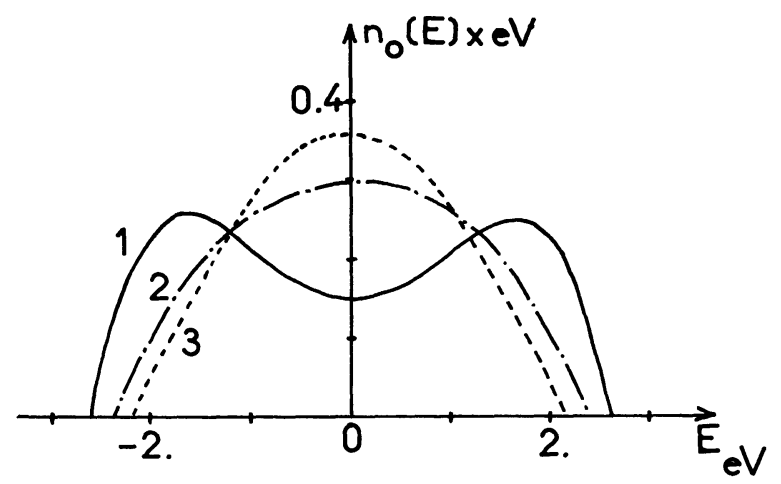

Fig. 1. - Approximate local impurity of states for a body centered cubic : 1) bulk atom;2) surface atom; 3) adatom on a (100) surface.

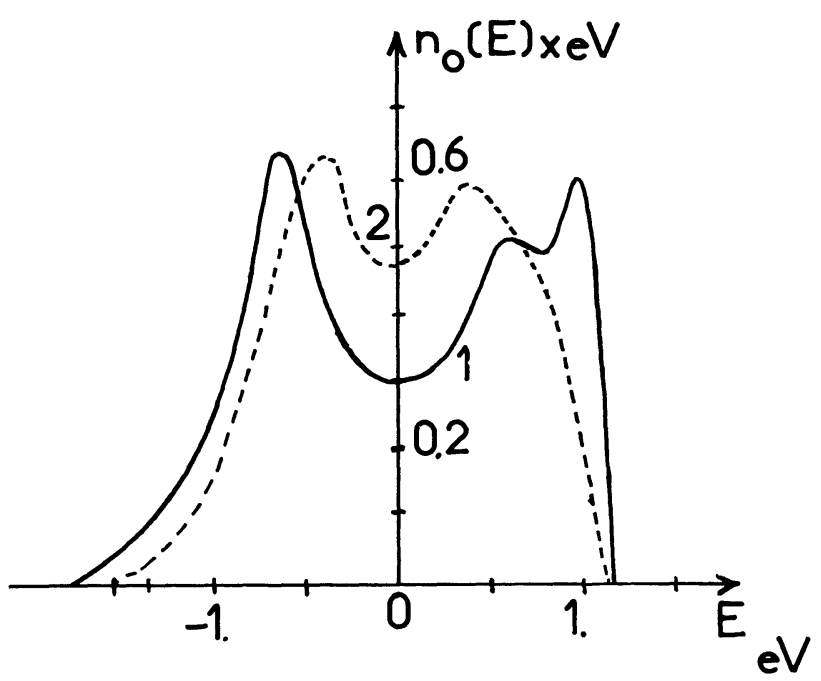

Fig. 2. - Local density of states from the results of Haydock et al. : 1) bulk atom ; 2) surface atom. 
gives the tendencies of the density of states obtained by the most elaborate recursive method of Haydock et al. [15] (Fig. 2). The important feature is the filling up of the minimum at the middle of the $d$ band which is quite nicely reproduced by our model. This behaviour is due to the decrease of the ratio $\mu_{4} / \mu_{2}^{2}$ from 1.6 to 2.2 . The discrepancy between the widths of the $\mathrm{d}$ band of figures 1 and 2 is due to the fact that the band width of Pettiford [16] for $\mathrm{Ni}$ is almost equal to $3 \mathrm{eV}$ and we deal with a $6 \mathrm{eV}$ band width. While our model is very crude, it can nevertheless show clearly the effect of the local surroundings of an atom. This is why we need to study the magnetic moment of an iron atom inside the bulk metal, or in the surface plane or adsorbed on a surface. The parameters $A, B$ and $E_{0}$ of the state density are given in table III.

\section{TABLE III}

Parameters of the density of states. $E_{0}$ is given in $\mathrm{eV}, A$ and $B$ respectively in $\mathrm{eV}$ minus 5 and minus 3

\begin{tabular}{lccc} 
& \multicolumn{1}{c}{$A$} & $B$ & $\underline{E_{0}}$ \\
Bulk & $+\overline{0.016}$ & $-\overline{0.090}$ & 2.59 \\
Surface & +0.002 & +0.036 & 2.43 \\
Adatom & -0.004 & +0.096 & 2.21
\end{tabular}

3. Scattering of the d electrons by the iron impurity. - In this section we consider the scattering of the $d$ electrons by the iron impurity. We assume that there are seven $d$ electrons on the iron atom. The excess of charge produces a self-consistent perturbing potential which is assumed to be localized on the iron atom.

We then use the same scattering method as Wolff [5] and the Friedel sum rule to describe the influence of the impurity on the $d$ band. We assume in an Anderson model that the perturbing potential $V$ due to the impurity is localized on the iron atom and it has, in the basis of the $d$ atomic functions, only one matrix element not equal to zero $V_{0}$

$$
V_{0}=\langle 0|V| 0\rangle
$$

where $|0\rangle$ is a $\mathrm{d}$ atomic function centered on the transition atom we replace by the iron atom. The wave functions $\varphi_{k}$ are scattered by an impurity into $\psi_{k}$ states given by

$$
\psi_{k}=\varphi_{k}+G_{0}^{+} V \psi_{k}
$$

where $G_{0}^{+}$is the Green operator

$$
G_{0}^{+}=\left[E-H_{0}+i \varepsilon\right]^{-1} .
$$

It is obvious that $H_{0}$ is the hamiltonian of a perfect infinite transition metal or a transition metal with a (100) surface or of a transition metal with a (100) surface with an adatom on it respectively for the three cases we consider.
We introduce the phase shift $\eta(E)$ [17]

$$
\eta(E)=-\operatorname{Arg} \operatorname{det}\left(1-G_{0}^{+} V\right) .
$$

In the tight-binding approximation, the eigenfunctions $\varphi_{k}$ are expressed as linear combinations of the atomic functions; with the approximation of a localized potential $V_{0}$, we have :

$$
\eta(E)=-\operatorname{Arg}\left(1-G_{0}(E) V_{0}\right)
$$

where

$$
G_{0}(E)=\left\langle 0\left|G_{0}^{+}\right| 0\right\rangle=F(E)-i \pi n_{\mathrm{L}}(E)
$$

and

$$
F(E)=\int \frac{n_{\mathrm{L}}\left(E^{\prime}\right) \mathrm{d} E^{\prime}}{E-E^{\prime}}
$$

The phase shift gives the total variation of the number of states

$$
\Delta N(E)=\frac{10}{\pi} \eta(E)
$$

The Friedel sum rule is satisfied in the case of an iron atom with seven $d$ electrons if :

$$
\Delta N\left(E_{\mathrm{F}}\right)=7-N
$$

$N$ is the number of $\mathrm{d}$ electrons of the transition metal and $E_{\mathrm{F}}$ the corresponding Fermi level.

This gives us a value of $V_{0}$ such that

$$
\operatorname{tg} \frac{(7-N) \pi}{10}=\frac{\pi V_{0} n_{\mathrm{L}}\left(E_{\mathrm{F}}\right)}{1-V_{0} F\left(E_{\mathrm{F}}\right)} .
$$

The values of $V_{0}$ versus $N$ are given in figure 3 .

In the three cases considered, the variation of the electron charges and the potential extend over the nearest neighbours of the impurity. The localization

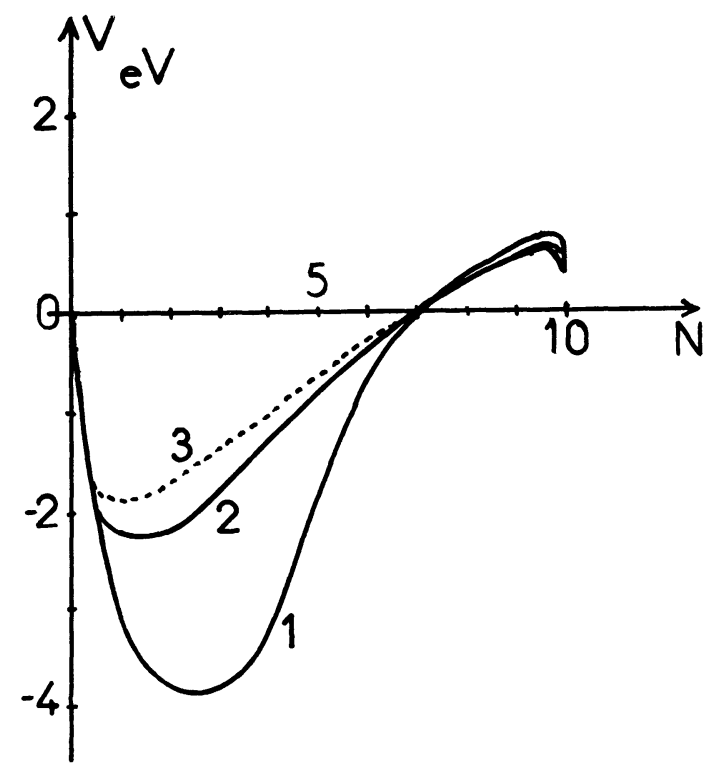

FIG. 3. - Self-consistent intraatomic potential : 1) bulk atom; 2) surface atom;3) adatom. 
of the potential is therefore a crude approximation. But if it seems necessary to take into account the matrix elements of the potential on the nearest neighbours of the impurity (Hubbard model) to calculate the binding energies of adatoms [18], [19], we may hope that their neglect does not change very much [20] the appearance of a magnetic moment, since the main potential is probably effectively localized on the impurity.

4. Local magnetic moment. - We consider now the cases when a magnetic instability may spread out. To do that we add an exchange term on the iron atom and we consider the local instability of the paramagnetic state of the iron atom.

Let us call $H_{\mathrm{m}}$ this exchange term :

$$
H_{\mathrm{m}}=U n_{\mathrm{d} \uparrow} n_{\mathrm{d} \downarrow}
$$

where $n_{\mathrm{d} \uparrow}$ and $n_{\mathrm{d} \downarrow}$ are respectively the local electronic density for the spin up and spin down bands.

A magnetic polarization appears if a local Stoner condition is satisfied [2] ... [6], [21], [22]

$$
5 U n_{\mathrm{L}}\left(E_{\mathrm{F}}\right) \geqslant 1
$$

where the factor 5 arises from the 5 degenerate bands and $n_{\mathrm{L}}\left(E_{\mathrm{F}}\right)$ is the local density of states on the iron atom which may be calculated from the perturbed Green operator $G^{+}$

$$
\begin{aligned}
G^{+} & =G_{0}^{+}+G_{0}^{+} V G^{+} \\
n_{\mathrm{L}}\left(E_{\mathrm{F}}\right) & \left.=-\frac{1}{\pi} \operatorname{Im}<0\left|G^{+}\right| 0\right\rangle .
\end{aligned}
$$

With our approximations, we find :

$$
n_{\mathrm{L}}\left(E_{\mathrm{F}}\right)=\frac{n_{0}\left(E_{\mathrm{F}}\right)}{\left[1-V_{0} F\left(E_{\mathrm{F}}\right)\right]^{2}+\pi^{2}\left[V_{0} n_{0}\left(E_{\mathrm{F}}\right)\right]^{2}} .
$$

The minimum value $U_{\mathrm{m}}$ of the exchange integral $U$ which is necessary to obtain a magnetic polarization is given by :

$$
U_{\mathrm{m}}=\frac{1}{5 n_{\mathrm{L}}\left(E_{\mathrm{F}}\right)}
$$

Figure 4 shows $U_{\mathrm{m}}$ versus the number of d electrons of the transition metal. If we look at the experimental results for a bulk iron impurity [1], we see that a magnetic polarization occurs for $N \geqslant 5.5$, which gives (Fig. 4, curve 1) $U_{\mathrm{m}}=1.4 \mathrm{eV}$. If we choose for the two other cases $U_{\mathrm{m}}=1.4 \mathrm{eV}$ which is a reasonable value for an iron atom in a bulk transition metal [12],

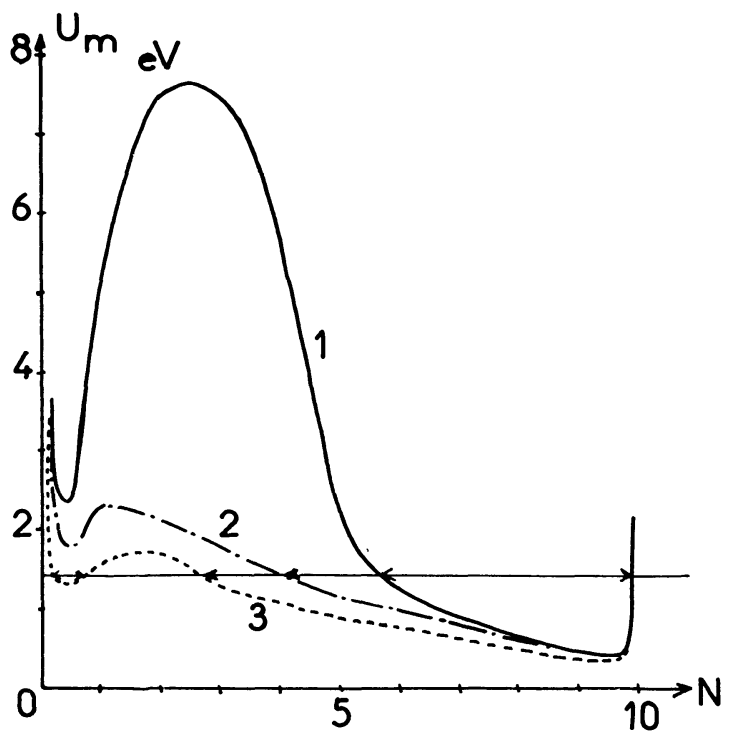

FIG. 4. $-U$ minimum for a magnetic polarization : 1) bulk atom ; 2) surface atom ; 3) adatom.

we see (Fig. 4, curves 2 and 3) that a magnetic moment appears for smaller $N$ in the case of a surface atom and still smaller for an adatom than for a bulk atom.

We think this result has some validity in spite of the approximations. This model may be adjusted to deal with other transitional serials taking into account the variation of the band-width. It will be interesting to have some experimental results in the case of a surface atom or an adatom. Probably a source experiment in Mössbauer effect will give us information on the magnetic polarization of the iron atoms [23]. Such experimental results, especially for the second transition row, are awaited for a more elaborate theory.

5. Conclusion. - A very simple model has been developed to study the appearance of a magnetic moment on an atom of iron. Three cases have been considered for the position of the atom of iron : substitutional in the bulk, on a surface position and in an adatom position. It has been shown that the magnetic polarization appears for a filling up of the $\mathrm{d}$ band smaller for a surface position than for a bulk position, and still smaller for an adatom position. Experimental results are awaited for an a more elaborate theory.

Acknowledgments. - We wish to thank Professor J. Friedel for his helpful advices.

\section{References}

[1] Clogston, A. M., Matthias, B. T., Peters, M., Williams, H. J., Corenzwit, E. and Sherwood, R. C., Phys. Rev. 125 (1962) 541.

[2] Friedel, J., Nuovo Cimento, Suppl. 7 (1958), 287, J. Phys. Rad. 23 (1962) 501.

[3] Blandin, A., Thèse Paris (1961), J. Appl. Phys. 39 (1968) 1285.
[4] Anderson, P. W., Phys. Rev. 124 (1961) 41.

[5] Wolff, P. A., Phys. Rev. 124 (1961) 1030.

[6] For a good review, see Heeger, A. J., Solid State Physics 23 (1969) 283.

[7] Slater, J. C., Phys. Rev. 49 (1936) 537, 931

[8] Kondo, J. Prog. Theor. Phys. (Kyoto) 32 (1964) 37, Solid State Physics 23 (1969) 181. 
[9] Slater, J. C. and Koster, G., Phys. Rev. 94 (1954) 1498. [10] Ducastelle, F. and Cyrot-Lackmann, F., J. Phys. \& Chem. Solids 31 (1970) 1295.

[11] Mattheiss, L. F., Phys. Rev. 139A (1965) 1893.

[12] Lannoo, M. and Allan, G., J. Phys. \& Chem. Solids 32 (1971) 637

Friedel, J., The Physics of Metals, ed. Ziman (Cambridge University Press) 1969, 340-408.

[13] Cyrot-Lackmann, F., Adv. Phys. 16 (1967) 393, J. Phys. \& Chem. Solids 29 (1968) 1235.

[14] Kalkstein, D. and Soven, P., Surf. Science 26 (1971) 85.

[15] Haydock, R., Heine, V. and Kelly, M. J., J. Phys. C 5 (1972) 2845

Haydock, R., Heine, V., Kelly, M. J. and Pendry, J. B., Phys. Rev. Lett. 29 (1972) 868.
[16] Pettifor, D. G., J. Phys. C 2 (1969) 1051, C 3 (1970) 367.

[17] Toulouse, G., Solid State Commun. 4 (1966) 593.

[18] Allan, G. and Lenglart, P., J. Physique 31 (1970) Supp. C4, C1-93.

[19] ThORPE, B. J., Surf. Science 33 (1972) 306.

[20] Gautier, F. and Lenglart, P., Phys. Rev., 139A (1965) 705.

[21] Stoner, E. C., Phil. Mag. 15 (1933) 1018, Proc. R. Soc. A 154 (1936) 656, A 165 (1938) 372, A 169 (1938) 339.

[22] Wohlfarth, E. P., Rev. Mod. Phys. 25 (1953) 211.

[23] Janot, C., Private communication.

Shinjot, T., Matsuzawa, T., Takada, T., Nasu, S. and Murukami, Y., Phys. Lett. A 36 (1971) 489.

[24] Wohlfarth, E. P., Proceedings of the international conference on Magnetism, Nottingham (1964) 51. 